\title{
Compositional Verification by Model Checking for Counter-Examples
}

\author{
Tevfik Bultan \\ Department of Computer Science \\ University of Maryland \\ College Park, MD 20742
}

\author{
Jeffrey Fischer \\ Rational Software Corporation \\ 8000 Westpark Drive \\ McLean, VA 22070
}

\author{
Richard Gerber \\ Institute for Advanced Computer Studies \\ Department of Computer Science \\ University of Maryland \\ College Park, MD 20742
}

\begin{abstract}
Many concurrent systems are required to maintain certain safety and liveness properties. One emerging method of achieving confidence in such systems is to statically verify them using model checking. In this approach an abstract, finite-state model of the system is constructed; then an automatic check is made to ensure that the requirements are satisfied by the model. In practice, however, this method is limited by the state space explosion problem.

We have developed a compositional method that directly addresses this problem in the context of multi-tasking programs. Our solution depends on three key space-saving ingredients: (1) checking for counter-examples, which leads to simpler search algorithms; (2) automatic extraction of interfaces, which allows a refinement of the finite model - even before its communicating partners have been compiled; and (3) using propositional "strengthening assertions" for the sole purpose of reducing state space.

In this paper we present our compositional approach, and describe the software tools that support it.
\end{abstract}

\section{Introduction}

Computing systems are now used in applications like automobiles, aircraft, and assembly lines. These systems often have safety-critical requirements; the computation must always produce the right answer. The computations typically require the coordinated efforts of multiple concurrent tasks. Critical properties, such as mutual exclusion and freedom

This work was supported in part by ONR grant N00014-94-10228, NSF grant CCR-9209333, and NSF Young Investigator Award CCR9357850 from deadlock, must be maintained by these tasks, but the temporal nature of these properties makes checking them a highly complex problem. This is particularly true when the properties depend on sequences of subtle task interactions. In such cases, some form of static analysis can often help a designer verify that the program will behave as expected.

Our general paradigm is this: to abstract our systems into mathematical models; to characterize specifications in terms of mathematical properties imposed on models; and to verify that models possess these properties. There is a wide range in capability and accuracy of various modeling techniques, as well as in the complexity of verifying properties of the models $[7,14,16]$. The particular technique we use is model checking.

Model checking refers to a family of algorithms used to check whether a state-transition structure satisfies a formula in some temporal logic. Several such logics [9, 13] and procedures $[6,10,15]$ have been proposed for this use. Our work uses CTL ${ }^{1}$ model checking [4], which runs in time linear in both the size of the structure and the length of the formula being verified.

The size of the structure normally dictates the cost of verification. When $n$ models of size $m$ are composed in parallel, the result can be a model of size $m^{n}$. This exponential blow up is commonly referred to as the state space explosion problem.

A considerable amount of research has been directed towards techniques for reducing the size of the model that gets constructed while applying model checking. Reduction techniques have generally followed one of two paths: (1) to avoid generating the entire structure, and instead to perform the check compositionally or locally [5, 8, 17, 18]; or (2) to form the whole structure, but encode it symbolically to reduce its size [2].

We have taken the compositional approach, albeit with several unique twists.

\footnotetext{
${ }^{1}$ Computation Tree Logic - a branching time temporal logic.
} 
First, we negate a user-entered invariant, and then search for its counter-example. We show how this type of query leads to a sound and complete theory of compositional model-checking, the preliminary sketch of which was first reported in [11] (which in turn extended the hardware analysis work in [3]).

Second, we exploit Ada's rendezvous-style communication, and automatically extract "logical interfaces" for each task. This allows us to refine a task's model with respect to its communicating partners - even before their models have been generated.

Third, we show how simple, "strengthening assertions" can be used to reduce the cost of verification.

Finally, we have developed a Tcl/Tk-driven toolset, in which each phase of model-checking is implemented as a $\mathrm{C}++$ class. In this manner, we have been able to measure the effects of various orderings of operations (e.g., compilation, composition, model-checking, etc.). We show some of these measurements in this paper.

The remainder of the paper is organized as follows. In Section 2 we motivate our compositional approach, and in Section 3 we give a more technical development of the theory, specially as it relates to verifying Ada programs. In Section 4 we show how our tool is used to support our approach, and in Section 5 we present some measurements of its effectiveness. We also overview some of the follow-on work we plan to pursue. We conclude in Section 6 with some remarks on the usefulness of this technology.

\section{Overview of the Solution}

Model checking requires two ingredients: a set of models (e.g., transition systems) and a set of properties expressed in temporal logic. Transition systems are generated from the source code of the program to be verified, each of which represents a task in the concurrent program. In our current implementation we concentrate on Ada programs. As the rest of this section shows, our method is general, and can be applied to any concurrent programming language.

The domain of the properties we are interested in includes two categories: safety and liveness. Safety properties express invariants of the system's behavior, i.e., they capture conditions that are expected to hold in each state of the system. A liveness property expresses a constraint on the future behavior of the system, i.e., that the current state of the system always leads-to an expected state.

After compiling the source code of the input program to the set of transition systems $T_{i}, T_{j}, \ldots$ a parallel composition, $T_{i}\left\|T_{j}\right\| \ldots$, can be formed, representing the concurrent executions of the set of transition systems. The problem of verifying that the concurrent program satisfies the given property is reduced to deciding whether or not the composite transition system satisfies $F$, where $F$ is the temporal logic formula representing the given property. I.e., if $T_{i}\left\|T_{j}\right\| \ldots=F$ then the property holds for the program.

Compositional approaches usually share a common objective - to solve $n$ individual problems of size $m$, as opposed to one problem of size $m^{n}$. For several very basic reasons, this goal has generally eluded researchers in the area of automated finite-state analysis. Consider the mechanics of model checking, where one uses a function "check(T : model, F : formula) : model," and expects it to return the sub-model of $T$ satisfying the formula $F$. In most cases the property $F$ is an "invariant" - which in CTL notation is written $\forall \square G$ for some formula $G$. When $\forall \square G$ is a requirement of a system $T$, we want $G$ to hold in all of $T$ 's states.

But when $T$ is the cartesian product of two models $T_{i}$ and $T_{j}$, we have the following:

\section{$T_{i} \| T_{j} \quad$ satisfies $\forall \square G$ iff $\operatorname{check}\left(T_{i} \| T_{j}, \forall \square G\right)=T_{i} \| T_{j}$.}

And herein lies the problem with compositional analysis. Since $T_{i}$ and $T_{j}$ interact with each other, if we wish to determine whether $\forall \square G$ holds, we will still end up generating the composite machine $T_{i} \| T_{j}$. When several concurrent models are involved, this technique can easily lead to "state space explosion."

Our approach is compositional, albeit at the expense of some generality. We restrict our input requirements to the common "global" ones; i.e., of the form $\forall \square G$ ("in every state $G$ holds"), $\forall \diamond G$ ("every trace contains a state where $G$ holds"), and $\forall \bigcirc G$ (" $G$ holds in all next states"). Then we achieve compositionality by taking a formula-dependent approach to minimizing each model.

Our algorithm essentially turns a verification query into its dual, and it progressively shrinks each model based on the (negated) requirements formula (see Figure 1). For example, assume we wish to determine whether $\forall \square G$ is true for $T_{i} \| T_{j}$. Then we have:

$$
\begin{array}{ll}
\operatorname{check}\left(T_{i} \| T_{j}, \forall \square G\right)=T_{i} \| T_{j} \quad & \text { iff } \\
& \operatorname{check}\left(T_{i} \| T_{j}, \exists \diamond \neg G\right)=\emptyset .
\end{array}
$$

The formula " $\exists \diamond \neg G$ " means "in some reachable state $\neg G$ holds;" this is true if and only if $G$ is not an invariant. Our 


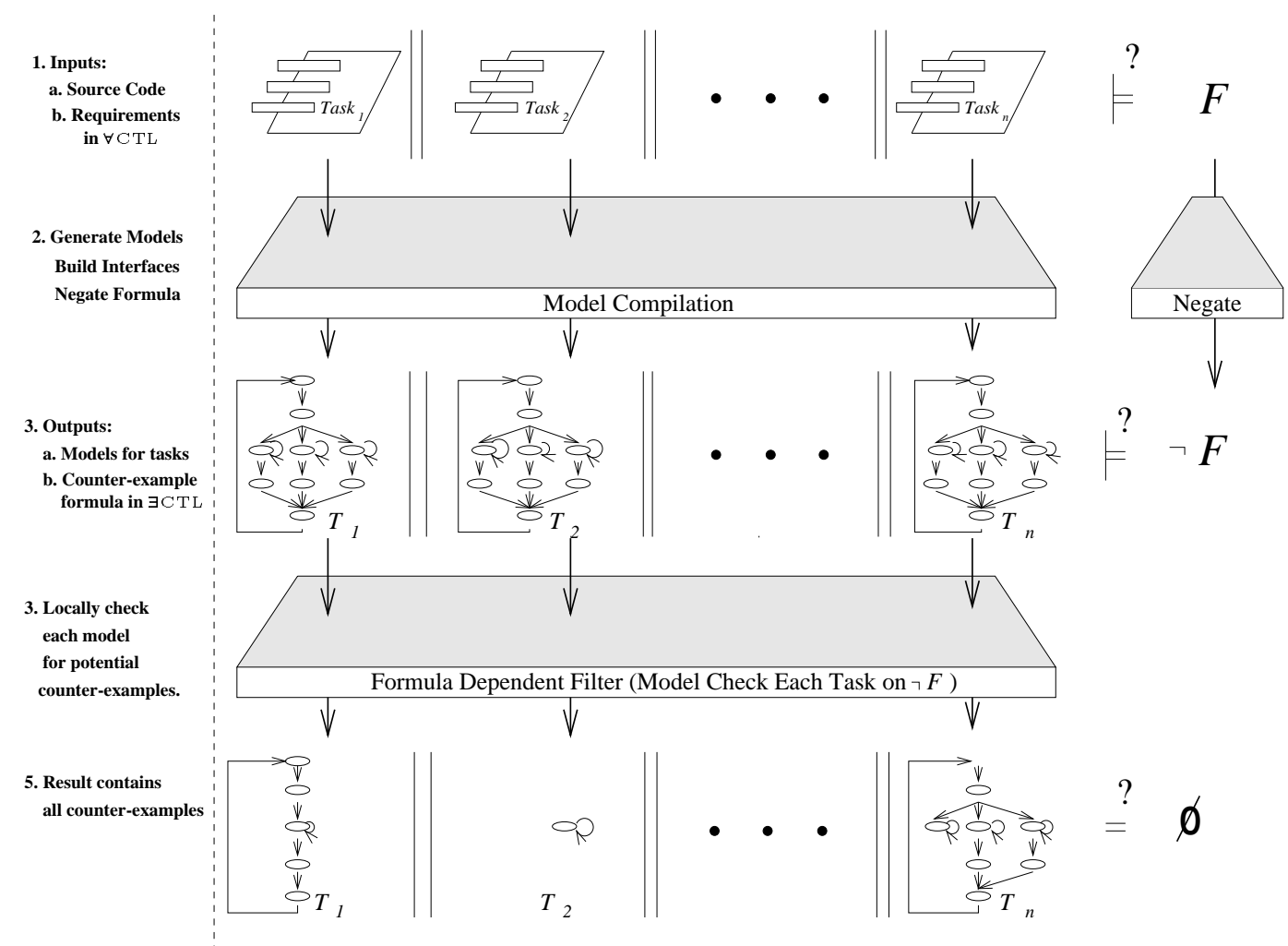

Figure 1: Our compositional verification method.

compositional checker check exploits simple facts like these, and hunts for "local" counter-examples to the input formula. Formally, the compositional result is:

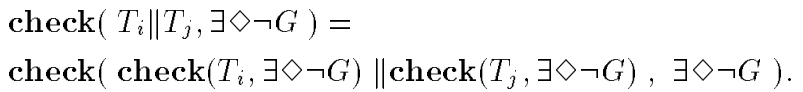

The right-hand side will often be considerably less expensive, since the inner calls to check conservatively delete transitions known not to be involved in any counter-examples. Thus the final composite contains all potential counterexamples - but nothing else.

\section{The Solution - Theory and Practice}

In this section we describe the steps of our method in detail, by way of a running example.

\subsection{An Example}

Consider the Ada tasks shown in Figure 2(top), where a program is composed of $n(n \geq 3)$ of these tasks. The $n-2$ middle tasks have a common structure (and differ only in their communications partners), while the 1 st and $n$th are unique.

\begin{tabular}{lll|}
\hline$F$ & $::=$ & $A|\forall \bigcirc F| \forall \square F|\forall \diamond F| F \forall \mathcal{U} F|F \wedge F| F \vee F$ \\
$A$ & $::=$ & $\langle$ atomic proposition $\rangle|\neg A| A \wedge A \mid A \vee A$ \\
\hline
\end{tabular}

Table 1: $\forall$ CTL.

As can be seen in Figure 2(bottom), we model an Ada program of $n$ tasks as a product of $n$ finite state machines. We abstract away data values and their influence on control flow. This allows us to concentrate on the task interaction, which is our chief concern - and it also keeps the associated decision problem tractable. Of course the price we pay may be a rather pessimistic analysis.

One requirement of our program may be the following property:

Whenever task $P_{i, i<n}$ has terminated, task $P_{i+1}$ will eventually terminate.

At the "user input level," this type of property is entered as a sentence in the universally quantified CTL (called $\forall$ CTL), a grammar for which is presented in Table 1 . In Table 2 we present the interpretation for the full CTL, i.e., the logic with both universal and existential quantifiers.

Using $\forall \mathrm{CTL}$, and the annotated labels in Fig- 


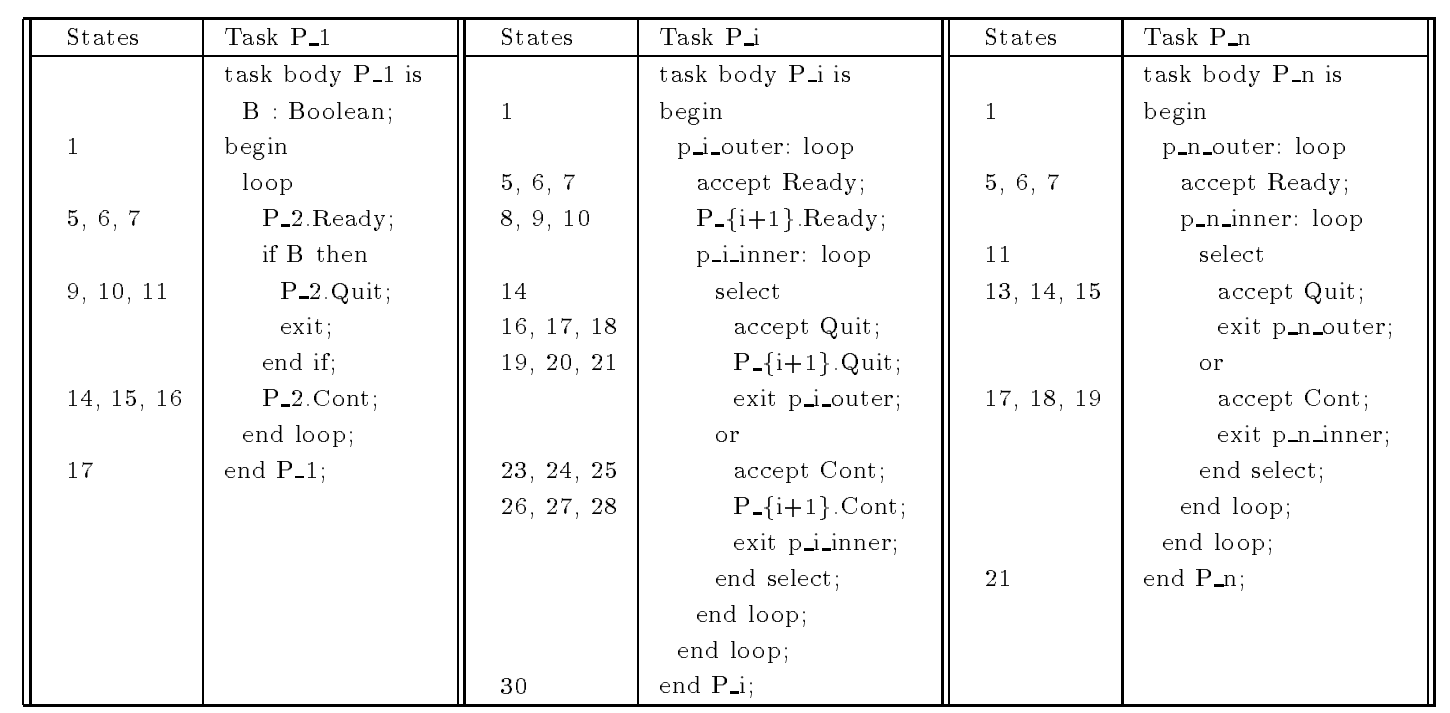

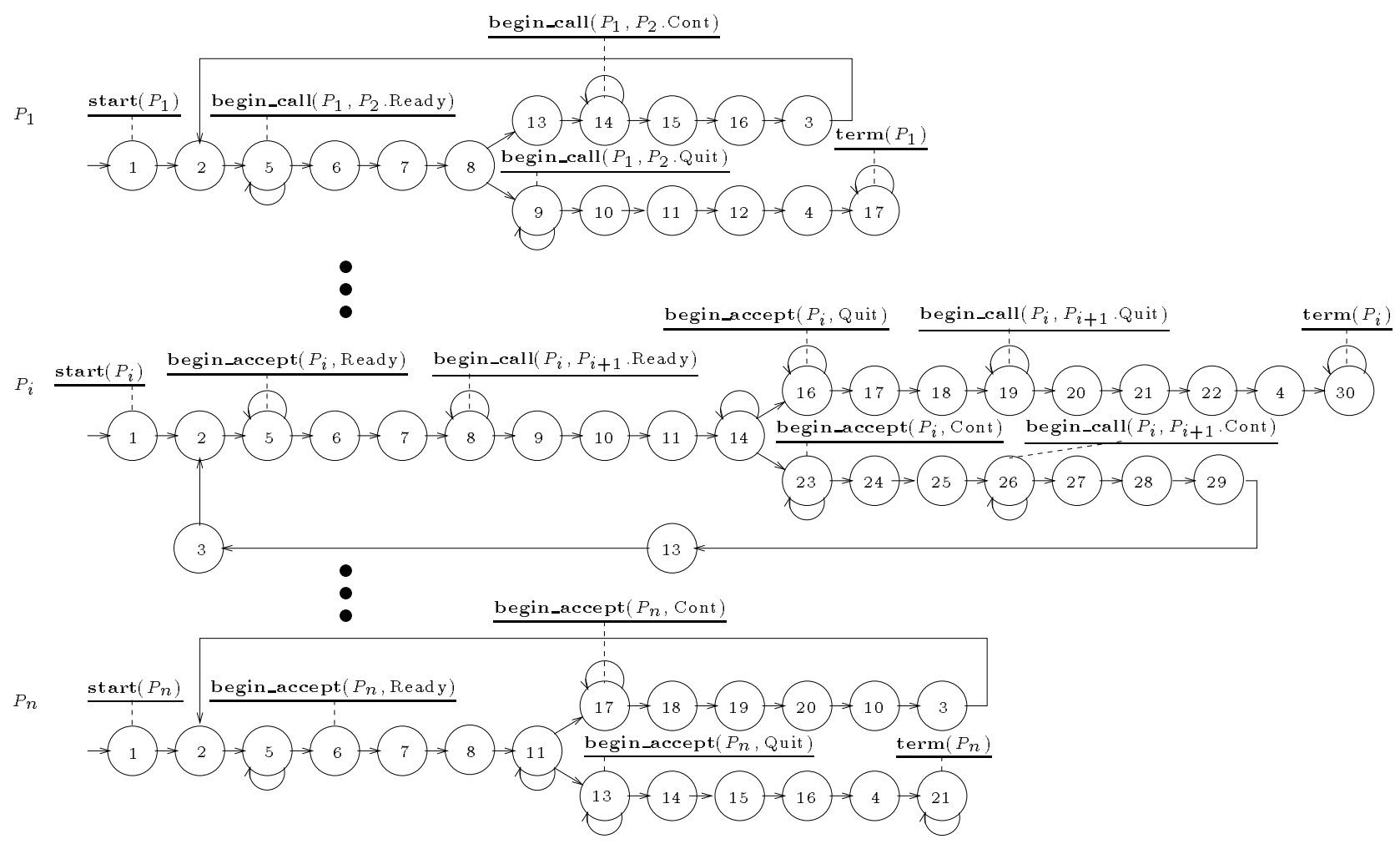

Figure 2: Source code (top), and transition relation graphs of $n$ task chain example (bottom). 


\begin{tabular}{|l|l|}
\hline Our Notation & Interpretation \\
\hline$f$ & $\begin{array}{l}f \text { is true in current state } \\
\text { for atomic proposition } f .\end{array}$ \\
$\forall \bigcirc F(\exists \bigcirc F)$ & $F$ is true on all (some) next states. \\
$\forall \diamond F(\exists \diamond F)$ & $F$ is eventually true on all (some) paths. \\
$\forall \square F(\exists \square F)$ & $F$ is invariantly true on all (some) paths. \\
$F \forall \mathcal{U} G(F \exists \mathcal{U} G)$ & $\begin{array}{l}\text { On all (some) paths } G \text { holds at some state } \\
\text { and } F \text { holds in every state on the path } \\
\end{array}$ \\
& prior to that state. \\
\hline
\end{tabular}

Table 2: CTL temporal operators.

\begin{tabular}{|lll|}
\hline$\neg \forall \square F$ & $\equiv$ & True $\exists \mathcal{U} \neg F$ \\
\hline$\neg \forall \diamond F$ & $\equiv$ & $\exists \square \neg F$ \\
\hline$\neg \forall \bigcirc F$ & $\equiv$ & $\exists \bigcirc \neg F$ \\
\hline$\neg\left(F_{1} \forall \mathcal{U} F_{2}\right)$ & $\equiv$ & $\left(\exists \square \neg F_{2}\right) \vee\left(\neg F_{2} \exists \mathcal{U}\left(\neg F_{1} \wedge \neg F_{2}\right)\right)$ \\
\hline
\end{tabular}

Table 3: CTL equivalences.

ure 2(bottom), our example's termination requirement can more formally be represented as follows:

$$
F \equiv \bigwedge_{i=1}^{n-1} \operatorname{term}\left(P_{i}\right) \leadsto \operatorname{term}\left(P_{i+1}\right)
$$

Here we use the familiar leads - to operator ("ح"), which is defined in $\forall \mathrm{CTL}$ as

$$
\mathcal{G}_{1} \leadsto \mathcal{G}_{2} \stackrel{\text { def }}{=} \forall \square\left(\mathcal{G}_{1} \Longrightarrow \forall \diamond\left(\mathcal{G}_{2}\right)\right) .
$$

One way or another, verifying this property entails: (1) considering the set of all states in which $P_{i}$ terminates, and (2) determining whether every reachable path leaving those states contains a state where task $P_{i+1}$ terminates.

The way we go about doing this is by converting the $\forall$ CTL properties into duals, and then checking for counterexamples. We present the rules for such a conversion in Table 3. As can be seen from the table, the dual of a $\forall \mathrm{CTL}$

\begin{tabular}{|l|l|}
\hline \multicolumn{2}{|l|}{$\begin{array}{l}\text { Satisfaction of an } \exists \text { CTL sentence, } F \text {, by state, } \vec{x}, \\
\text { wrt. } \Sigma \text {, the set of paths in the model. }\end{array}$} \\
\hline$F \equiv \exists \bigcirc G$ & $\vec{x} \models F$ iff $\exists \sigma \in \Sigma:$ \\
& $\vec{x}=\sigma(0) \wedge \sigma(1) \models G$ \\
\hline$F \equiv \exists \diamond G$ & $\vec{x} \models F$ iff $\exists \sigma \in \Sigma:$ \\
& $\vec{x}=\sigma(0) \wedge(\exists j \geq 0: \sigma(j) \models G)$ \\
\hline$F \equiv \exists \square G$ & $\vec{x} \models F$ iff $\exists \sigma \in \Sigma:$ \\
& $\vec{x}=\sigma(0) \wedge(\forall j \geq 0: \sigma(j) \models G)$ \\
\hline$F \equiv G \exists \mathcal{U} H$ & $\vec{x} \models F$ iff $\exists \sigma \in \Sigma:$ \\
& $\vec{x}=\sigma(0) \wedge(\exists j \geq 0: \sigma(j) \models H \wedge$ \\
& $\forall k: 0 \leq k<j: \sigma(k) \models G)$ \\
\hline
\end{tabular}

Table 4: $\exists$ CTL logic semantics. formula will always end up in $\exists$ CTL, i.e., the existentially quantified sublanguage of CTL. The semantics of the $\exists \mathrm{CTL}$ temporal operators is given in Table 4.

Consider one of the conjuncts of our requirement $F$ :

$$
F_{i} \equiv \operatorname{term}\left(P_{i}\right) \sim \operatorname{term}\left(P_{i+1}\right)
$$

which possesses as its dual the $\exists$ CTL sentence:

$$
\neg F_{i} \equiv \exists \diamond\left(\operatorname{term}\left(P_{i}\right) \wedge \exists \square \neg \operatorname{term}\left(P_{i+1}\right)\right)
$$

Let $\overrightarrow{x_{I}}$ be the initial state of the model of our program, and let $\sigma(i)$ the $i$ th state in the path $\sigma$. Then in the positive (universally quantified) logic, we are asking the question:

$$
\forall \sigma: \sigma(0)=\overrightarrow{x_{I}}: \sigma \models F_{i} ?
$$

But instead, we will ask whether there are any counterexamples:

$$
\exists \sigma: \sigma(0)=\overrightarrow{x_{I}}: \sigma \models \neg F_{i} \quad ?
$$

This is often (though not always) a much less expensive question to answer.

\subsection{Models}

Consider a system of $n$ tasks, in which the states of task $i$ are drawn from a finite set

$$
S_{i}=\left\{s_{i_{1}}, s_{i_{2}}, \ldots, s_{i_{m}}\right\}
$$

where each $s_{i_{j}}$ represents a potential control point in task $i$. On the other hand, a state in the full composition of all of the tasks will range over

$$
S=S_{1} \times S_{2} \times \ldots \times S_{n} .
$$

We denote elements of $S$ by vectors such as

$$
\overrightarrow{x_{j}}=\left(s_{1_{j 1}}, s_{2_{j 2}}, \ldots, s_{n_{j n}}\right)
$$

where each $s_{i_{j i}} \in S_{i}$.

The set of atomic propositions, AP, consists of state labels introduced automatically during the model generation process as well as user defined labels that denote specific states in the execution of a task. We define a set of valuation functions for $A P$ of the sort:

$$
f:\left(S_{1} \times S_{2} \times \ldots \times S_{n}\right) \rightarrow\{\text { True, False }\} .
$$

For each $f \in A P$ its complement $\neg f$ is also defined as an atomic proposition in $A P$ such that $\neg f(\vec{x})$ maps to True (False) if and only if $f(\vec{x})$ maps to False (True). 


\subsection{Transitions}

The global, closed system's transitions $T$ range over $S \times S$, where if $\left(\vec{x}, \vec{x}^{\prime}\right) \in T$, the interpretation is that the system can move from $\vec{x}$ to $\vec{x}^{\prime}$. We alternatively use the notation $\vec{x} \rightarrow \vec{x}^{\prime}$ to denote the fact that $\left(\vec{x}, \vec{x}^{\prime}\right) \in T$.

In practice, of course, we usually do not deal with global transitions - after all, we are aiming for a compositional strategy, where we can perform our analysis incrementally.

When task $i$ is compiled, its local transition model is formed, which we denote $\widehat{T}_{i} \subseteq S_{i} \times S_{i}$. This model is generated independently of the others, and an element $\left(s_{i}, s_{i}^{\prime}\right)$ of $\widehat{T}_{i}$ does not include any information about the states of the other tasks.

However we can (and do) give $\widehat{T}_{i}$ meaning in the closed system as well. The local transition system can trivially be extended into a set of potential global transitions as follows:

$$
\begin{array}{r}
T_{i}=\left\{\left(s_{1}, \ldots, s_{i}, \ldots, s_{n}\right) \rightarrow\left(s_{1}^{\prime}, \ldots, s_{i}^{\prime}, \ldots, s_{n}^{\prime}\right)\right. \\
\left.\mid\left(s_{i}, s_{i}^{\prime}\right) \in \widehat{T}_{i} \wedge \forall j: s_{j}, s_{j}^{\prime} \in S_{j}\right\} .
\end{array}
$$

Obviously we do not actually expand out this set $T_{i}$. Rather, when the analyzer generates a local state, it uses the "wild card" symbol ("-") to refer to the other parts of the system. For example, let $\left(s_{i}, s_{i}^{\prime}\right) \in \widehat{T}_{i}$, and consider the following symbolic transition:

$$
\left(-, \ldots,-, s_{i},-, \ldots,-\right) \rightarrow\left(-, \ldots,-, s_{i}^{\prime},-, \ldots,-\right)
$$

We use this to denote the set of all potential global transitions consistent with $\left(s_{i}, s_{i}^{\prime}\right)$, i.e.,

$\left\{\left(s_{1}, \ldots, s_{i}, \ldots, s_{n}\right) \rightarrow\left(s_{1}^{\prime}, \ldots, s_{i}^{\prime}, \ldots, s_{n}^{\prime}\right) \mid \forall j: s_{j}, s_{j}^{\prime} \in S_{j}\right\}$

As we go about composing transition systems, and performing model-checking on them, we refine these wild cards into real system states.

\subsection{Composition: Theory and Practice}

If two transition models, $T_{i}$ and $T_{j}$, were completely independent, then getting their parallel composition would simply be a matter of taking their intersection, $T_{i} \cap T_{j}$. But in general, concurrent tasks are not independent - they share synchronization and communication constraints. We capture this by a set of $\forall \mathrm{CTL}$ formulae, the conjunction of which we call a refinement relation. A decision procedure "chops off" transitions inconsistent with the refinement relation, i.e., those not allowed by the language's semantics. The refinement relation itself is generated by the compiler; it uses the source program and a set of target-specific semantic rules to form each clause.

We demonstrate this concept on our example program. In Figure 3 we give a more detailed transition graph for task $P_{1}$. The labels on the states are atomic propositions, which are generated by the compiler. The interpretations for some of these atoms are as follows:

start(task): true in task's initial state.

begin_call(task,entry): true in the states where task is ready to begin a call on the specified entry.

active_call(task, entry): true in the states where task's call to the specified entry has been accepted.

end_call(task,entry): true in the states where task's call to the specified entry has just completed.

term(task): true in the state associated with task's termination.

Consider the "Ready" rendezvous between tasks $P_{1}$ and $P_{2}$. If $P_{1}$ and $P_{2}$ are both prepared to rendezvous, then they will be in states labeled begin_call( $P_{1}, P_{2}$. Ready) ( $P_{1}$ 's state 5$)$, and begin_accept $\left(P_{2}\right.$, Ready) ( $P_{2}$ 's state 5$)$, respectively. The rendezvous occurs when $P_{1}$ and $P_{2}$ take the transitions to the states labeled active_call $\left(P_{1}, P_{2}\right.$. Ready) ( $P_{1}$ 's state 6$)$, and active_accept $\left(P_{2}\right.$, Ready) ( $P_{2}$ 's state 6$)$, respectively. Ada's semantics insist that the rendezvous will occur if and only if both tasks commit to it, i.e., if and only if both tasks take their local transitions $5 \rightarrow 6$.

But the intersection of these two models includes transitions that are counter to the semantics, e.g.:

$$
(5,5, \ldots) \rightarrow(6,5, \ldots) \in T_{1} \cap T_{2}
$$

That is, $P_{1}$ autonomously decides to rendezvous whereas $P_{2}$ decides to wait. Such a transition is deleted in our refinement check.

The refinement relation is built by doing a simple scan of all the tasks in the program, and extracting their inter-task interfaces. Then, from the interface data, the analyzer determines which tasks synchronize with which other tasks, and which propositions must hold when such synchronizations occur.

The entire relation, $\mathcal{R}$ is specified via a CTL formula. The CTL formula specifying $\mathcal{R}$ is the conjunction of subformulae in the form $f \rightarrow g$ or $f \rightarrow \forall \bigcirc(g)$, where $f$ and $g$ are propositions containing only the atomic propositions 


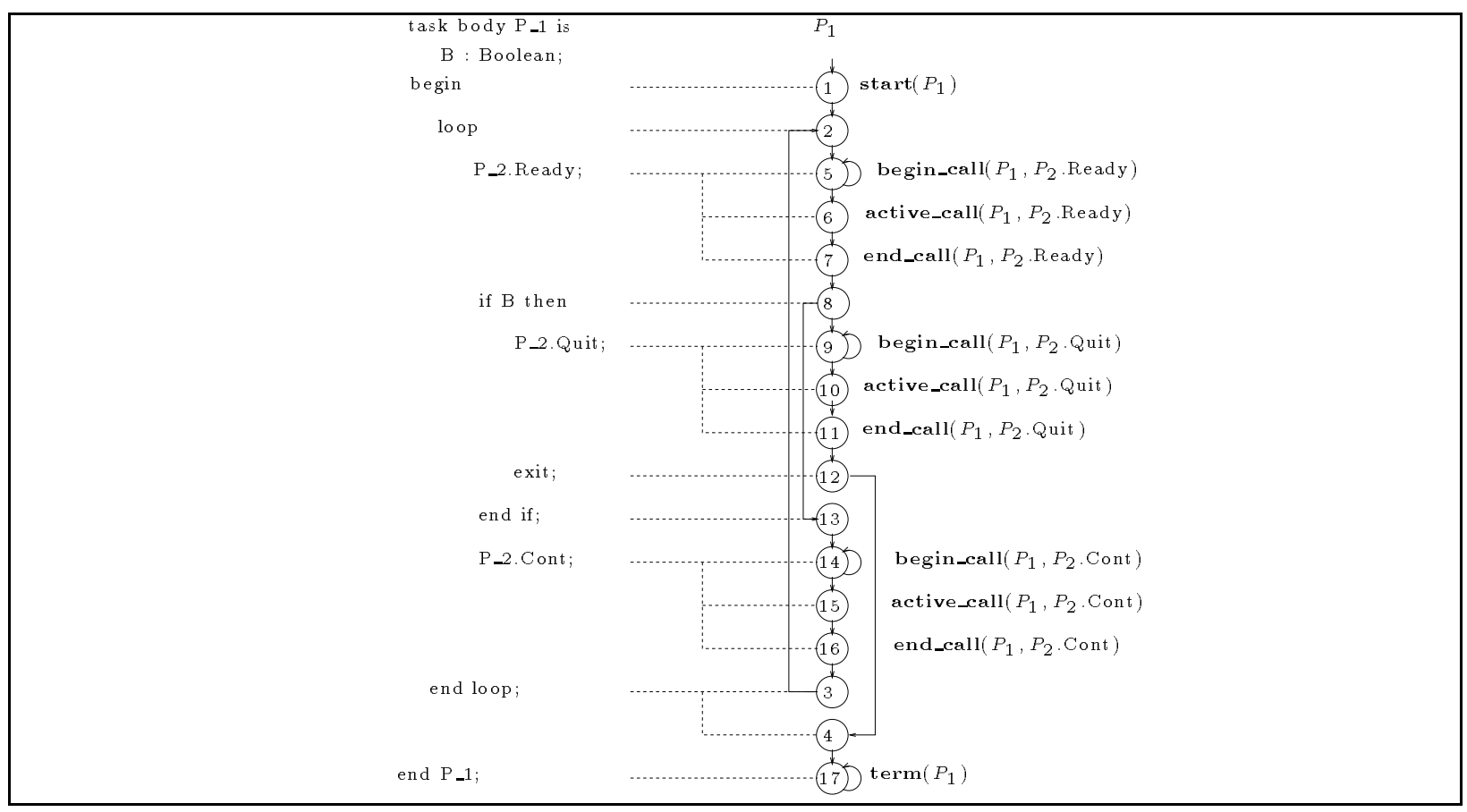

Figure 3: Source code and transition relation graph of the first task in the $n$ task chain example.

and boolean connectives. In Figure 4, we show the part of $\mathcal{R}$ which refines the transition systems with respect to task $P_{i}$. The complete refinement relation will be the conjunction of similar formulae for each task. Explanations for two of the subformulae (annotated in Figure 4) are given below:

1 If $P_{i-1}$ is ready to call "P_i.Ready" and if $P_{i}$ is ready to "accept Ready," then in all next states both $P_{i-1}$ and $P_{i}$ are in the rendezvous.

2 If task $P_{i}$ is in rendezvous "P_i+1. Ready," then task $P_{i+1}$ must be in the rendezvous at its "accept Ready."

The parallel composition of $T_{i}$ and $T_{j}$ can be defined as follows:

$$
T_{i} \| T_{j} \stackrel{\text { def }}{=} T_{i} \cap T_{j} \cap T_{\mathcal{R}}
$$

where $T_{\mathcal{R}}$ is the largest set of transitions satisfying $\mathcal{R}$. The algorithm in Figure 5 effectively computes this composed transition model.

\subsection{Model Checking}

Our compositional model-checker does not try to make exact decisions about a property (which usually requires a complete model). Rather, it incrementally refines estimates using partial models. These estimates are conservative, in that if any counter-examples to the specified property exist then the partial models we return from our checks will always contain them. As a result, if any check on a partial model fails to produce a potential counter-example, then we can immediately infer that the complete model does not have any either. To decide the subset of a model that satisfies a formula we recursively apply the projection functions shown in Table 5. We note that while these functions yield estimates for partial compositions, e.g., $T_{i} \| T_{j}$, they give exact results when applied to a composition that includes all components, e.g., $T$.

Consider evaluating an atomic proposition on the transition relation $T_{p}$. Assume that $T_{p}$ is a partial model, i.e., $p \subset\{1, \ldots, n\}$. Then a state $\overrightarrow{x_{p}}$ of this model is of the form:

$$
\overrightarrow{x_{p}}=\left(s_{p_{1}}, \ldots, s_{p_{m}},-, \ldots,-\right)
$$

where $m$ is the cardinality of $p$. This model does not give us any information about the states of the tasks which are not in this partial model. The only information we have about the non-local models (i.e., the models which are not in set $p$ ) is the synchronization constraints defined by $\mathcal{R}$. So, it may be the case that when we try to evaluate an atomic proposition $f$ on $\overrightarrow{x_{p}}$, both $f\left(\overrightarrow{x_{p}}\right)$ and $\neg f\left(\overrightarrow{x_{p}}\right)$ are True. Consequently, the projection function could decide a transition, with $\overrightarrow{x_{p}}$ as its source state, potentially satisfies both $f$ and $\neg f$. Now 
Refinement Formula for Task $P_{i}$ (Imposes Ada Semantics)

$\left(\right.$ begin_call $\left(P_{i-1}, P_{i}\right.$. Ready $) \wedge$ begin_accept $\left(P_{i}\right.$, Ready $\left.)\right) \rightarrow \forall \bigcirc\left(\operatorname{active\_ call}\left(P_{i-1}\right.\right.$, Ready $\left.) \wedge \operatorname{active\_ accept}\left(P_{i}, \operatorname{Ready}\right)\right){ }^{\mathbf{T}}$

$\left(\right.$ begin_accept $\left(P_{i}\right.$, Ready $) \wedge \neg$ begin_call $\left(P_{i-1}, P_{i}\right.$.Ready $) \rightarrow \forall \bigcirc\left(\right.$ begin_accept $\left.\left(P_{i}, \operatorname{Ready}\right)\right)$

(active_call $\left(P_{i}, P_{i+1}\right.$. Ready) $\wedge$ active_accept $\left(P_{i+1}\right.$, Ready $\left.)\right) \rightarrow \forall \bigcirc\left(\right.$ end_call $\left(P_{i}, P_{i}+1\right.$. Ready $\left.)\right)$

(begin_call $\left(P_{i}, P_{i+1}\right.$.Ready) $\wedge \neg$ begin_accept $\left(P_{i+1}\right.$, Ready $\left.)\right) \rightarrow \forall \bigcirc\left(\right.$ begin_call $\left(P_{i}, P_{i+1}\right.$. Ready))

(begin_call $\left(P_{i-1}, P_{i}\right.$.Quit) $\wedge$ begin_accept $\left(P_{i}\right.$, Quit $\left.)\right) \rightarrow \forall \bigcirc\left(\operatorname{active\_ call}\left(P_{i-1}\right.\right.$, Quit $) \wedge \operatorname{active\_ accept}\left(P_{i}\right.$, Quit $)$ )

(begin_accept $\left(P_{i}\right.$, Quit) $\wedge \neg$ begin_call $\left(P_{i-1}, P_{i}\right.$.Quit $\left.)\right) \rightarrow \forall \bigcirc$ (begin_accept $\left(P_{i}\right.$, Quit))

(active_call $\left(P_{i}, P_{i+1}\right.$.Quit) $\wedge$ active_accept $\left(P_{i+1}\right.$, Quit $\left.)\right) \rightarrow \forall \bigcirc$ (end_call $\left(P_{i}, P_{i+1}\right.$. Quit $\left.)\right)$

(begin_call $\left(P_{i}, P_{i+1}\right.$. Quit) $\wedge \neg$ begin_accept $\left(P_{i+1}\right.$, Quit $\left.)\right) \rightarrow \forall \bigcirc$ (begin_call $\left(P_{i}, P_{i+1}\right.$. Quit))

(begin_call $\left(P_{i-1}, P_{i}\right.$.Cont $) \wedge$ begin_accept $\left(P_{i}\right.$, Cont $\left.)\right) \rightarrow \forall \bigcirc\left(\operatorname{active\_ call}\left(P_{i-1}\right.\right.$, Cont $) \wedge$ active_accept $\left.\left(P_{i}, \mathrm{Cont}\right)\right)$

(begin_accept $\left(P_{i}\right.$, Cont) $\wedge \neg$ begin_call $\left(P_{i-1}, P_{i}\right.$.Cont $\left.)\right) \rightarrow \forall \bigcirc$ (begin_accept $\left(P_{i}\right.$, Cont $)$ )

(active_call $\left(P_{i}, P_{i+1}\right.$. Cont $) \wedge \operatorname{active\_ accept}\left(P_{i+1}\right.$, Cont $\left.)\right) \rightarrow \forall \bigcirc$ (end_call $\left(P_{i}, P_{i+1}\right.$. Cont $)$ )

(begin_call $\left(P_{i}, P_{i+1}\right.$. Cont $) \wedge \neg$ begin_accept $\left(P_{i+1}\right.$, Cont $\left.)\right) \rightarrow \forall \bigcirc$ (begin_call $\left(P_{i}, P_{i+1}\right.$.Cont $)$ )

$\left(\operatorname{at\_ select}\left(P_{i}\right) \wedge\right.$ (begin_call $\left(P_{i-1}, P_{i}\right.$. Quit) $\vee$ begin_call $\left(P_{i-1}, P_{i}\right.$.Cont $\left.\left.)\right)\right) \rightarrow$

$\forall \bigcirc\left(\left(\right.\right.$ begin_accept $\left(P_{i}\right.$, Quit $) \wedge$ begin_call $\left(P_{i-1}, P_{i}\right.$.Quit $\left.)\right) \vee\left(\right.$ begin_accept $\left(P_{i}\right.$, Cont $) \wedge$ begin_call $\left(P_{i-1}, P_{i}\right.$.Cont $\left.)\right)$

(active_accept $\left(P_{i}\right.$, Ready $) \rightarrow \forall \bigcirc\left(\right.$ end_accept $\left(P_{i}\right.$, Ready $\left.)\right) \wedge \quad\left(\operatorname{active\_ accept}\left(P_{i}, \operatorname{Ready}\right) \rightarrow \operatorname{active\_ call}\left(P_{i-1}, P_{i}\right.\right.$. Ready $\left.)\right)$

(end_accept $\left(P_{i}\right.$, Ready $) \rightarrow$ end_call $\left(P_{i-1}, P_{i}\right.$.Ready $)$ )

end_call $\left(P_{i}, P_{i+1}\right.$.Ready $) \rightarrow \operatorname{end\_ accept}\left(P_{i+1}, \operatorname{Ready}\right) \wedge \operatorname{active\_ call}\left(P_{i}, P_{i+1}\right.$. Ready $\rightarrow \operatorname{active\_ accept}\left(P_{i+1}, \operatorname{Ready}\right) \mathbf{2}$

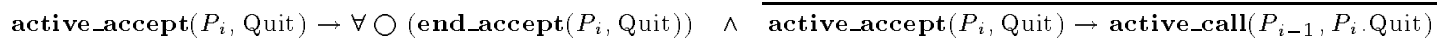

end_accept $\left(P_{i}\right.$, Quit $) \rightarrow \operatorname{end\_ call}\left(P_{i-1}, P_{i}\right.$.Quit $) \wedge \operatorname{begin\_ accept}\left(P_{i}\right.$, Quit $) \rightarrow \operatorname{begin\_ call}\left(P_{i-1}\right.$, Quit $)$

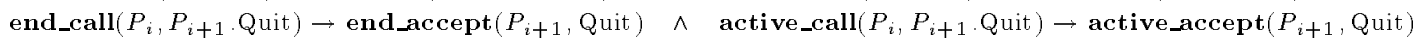

$\operatorname{active\_ accept}\left(P_{i}\right.$, Cont $) \rightarrow \forall \bigcirc\left(\right.$ end_accept $\left(P_{i}\right.$, Cont $\left.)\right) \wedge \operatorname{active\_ accept}\left(P_{i}\right.$, Cont $) \rightarrow \operatorname{active\_ call}\left(P_{i-1}, P_{i}\right.$. Cont $)$

end_accept $\left(P_{i}, \mathrm{Cont}\right) \rightarrow \operatorname{end} \_$call $\left(P_{i-1}, P_{i}\right.$.Cont $) \wedge \quad \operatorname{begin\_ accept}\left(P_{i}, \mathrm{Cont}\right) \rightarrow \operatorname{begin\_ call}\left(P_{i-1}, \mathrm{Cont}\right)$

end_call $\left(P_{i}, P_{i+1}\right.$.Cont $) \rightarrow \operatorname{end} \_\operatorname{accept}\left(P_{i+1}\right.$, Cont $) \wedge \operatorname{active\_ call}\left(P_{i}, P_{i+1}\right.$. Cont $) \rightarrow \operatorname{active\_ accept}\left(P_{i+1}\right.$, Cont $)$

Figure 4: Automatically generated refinement formula for task $P_{i}$.

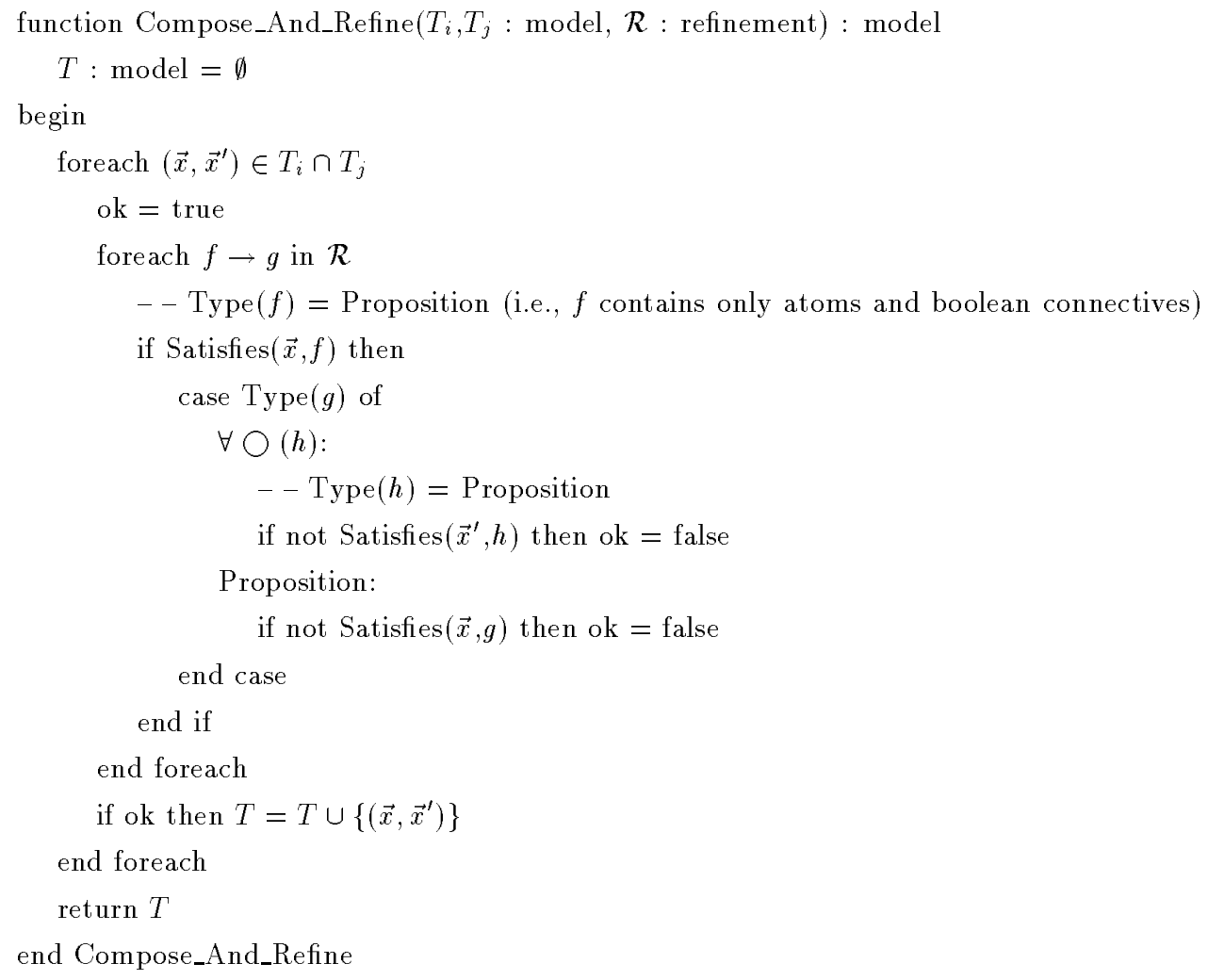

Figure 5: Parallel composition. 


\begin{tabular}{|c|l|}
\hline$\exists$ CTL Subformula $\mathcal{F}$ & projection $(T, \mathcal{F})$ \\
\hline$f \in A P$ & $\left\{\left(\overrightarrow{x_{1}}, \overrightarrow{x_{2}}\right) \in T \mid f\left(\overrightarrow{x_{1}}\right) \wedge\left(\overrightarrow{x_{1}}, \overrightarrow{x_{2}}\right) \in T_{\mathcal{R}}\right\}$ \\
\hline$f \wedge g$ & projection $(T, f) \cap$ projection $(T, g)$ \\
\hline$f \vee g$ & projection $(T, f) \cup$ projection $(T, g)$ \\
\hline$\exists \bigcirc f$ & $\left\{\left(\overrightarrow{x_{1}}, \overrightarrow{x_{2}}\right) \in T \mid \exists\left(\overrightarrow{x_{1}}, \overrightarrow{x_{3}}\right) \in T\right.$ \\
& $\wedge \exists\left(\overrightarrow{x_{3}}, \overrightarrow{x_{4}}\right) \in$ projection $\left.(T, f)\right\}$ \\
\hline$\exists \square f$ & $\left\{\left(\overrightarrow{x_{1}}, \overrightarrow{x_{2}}\right) \in T \mid \exists\left(\overrightarrow{x_{1}}, \overrightarrow{x_{3}}\right) \in\right.$ fix $\left.T_{n}\right\}$ \\
& with $f i x_{n} T_{n}: T_{0}=$ projection $(T, f)$ \\
& $T_{n+1}=\left\{\left(\overrightarrow{x_{1}}, \overrightarrow{x_{2}}\right) \in T_{n}\right.$ \\
& $\left.\mid \exists\left(\overrightarrow{x_{2}}, \overrightarrow{x_{3}}\right) \in T_{n}\right\}$ \\
\hline & $\left\{\left(\overrightarrow{x_{1}}, \overrightarrow{x_{2}}\right) \in T \mid \exists\left(\overrightarrow{x_{1}}, \overrightarrow{x_{3}}\right) \in\right.$ fix $\left.T_{n}\right\}$ \\
& with $f i x_{n} T_{n}: \overrightarrow{T_{0}}=$ projection $(T, g)$ \\
& $T_{n+1}=\left\{\left(\overrightarrow{x_{1}}, \overrightarrow{x_{2}}\right) \in\right.$ projection $(T, f)$ \\
& $\left.\mid \exists\left(\overrightarrow{x_{2}}, \overrightarrow{x_{3}}\right) \in T_{n}\right\} \cup T_{n}$ \\
\hline
\end{tabular}

Table 5: Projection functions for $\exists$ CTL.

assume that $T_{p}$ is not a partial model. Then it is the case that the state is completely specified:

$$
\overrightarrow{x_{p}}=\left(s_{1}, \ldots, s_{i}, \ldots, s_{n}\right)
$$

and so only one of $f$ and $\neg f$ can be true, and so in this case the projection function returns the exact result.

Another crucial point in the evaluation of atomic propositions on partial models is the usage of the refinement relation $\mathcal{R}$. As can be seen in the first row of Table 5, projection function of an atomic proposition $f$ includes transitions which satisfy two conditions: 1) $f$ evaluates to True for the source state and 2) the transition is consistent with the refinement relation. If we have a partial model $T_{p}$ as defined above, then these conditions may be satisfied by the transition $\left(\overrightarrow{x_{p}}, \overrightarrow{x_{p}}\right)$ by substituting non-local states for the don't care conditions "-". For example,

$$
\begin{array}{r}
f\left(\overrightarrow{x_{p}}\right)=\text { True iff } \exists s_{j_{1}}, \ldots, s_{j_{n-m}}: j_{1}, \ldots, j_{n-m} \notin p: \\
f\left(s_{p_{1}}, \ldots, s_{p_{m}}, s_{j_{1}}, \ldots, s_{j_{n-m}}\right)=\text { True. }
\end{array}
$$

The second condition requires that the same substitutions for the non-local states should be consistent with the refinement relation. Hence, the the complete condition is :

$$
\begin{aligned}
& \exists s_{j_{1}}, \ldots, s_{j_{n-m}}: j_{1}, \ldots, j_{n-m} \notin p: \\
& f\left(s_{p_{1}}, \ldots, s_{p_{m}}, s_{j_{1}}, \ldots, s_{j_{n-m}}\right)=\text { True } \wedge \\
& \left(\left(s_{p_{1}}, \ldots, s_{p_{m}}, s_{j_{1}}, \ldots, s_{j_{n-m}}\right), x_{p}^{\prime}\right) \in T_{\mathcal{R}} .
\end{aligned}
$$

This mechanism enables us to remove the transitions which are inconsistent with atomic proposition $f$ according to the refinement relation. We implement this as follows: when we are computing projection of an atomic proposition we pull out the subformulae of the refinement relation which involves that particular atomic proposition. We check if the local states of the transition are consistent with these subformulae.

The evaluation of the other projection functions is straight-forward. Consider the evaluation of a $\exists \square(f)$ formula wrt. the transition relation $T$. We first identify $T_{0} \subseteq T$ where $f$ holds in the source state. Then, from a set of transitions $T_{n-1}$, we construct a set $T_{n}$ by removing transitions with destination states that are not in $T_{n-1}$. Obviously the fixpoint is reached when deletion stops; i.e., when $T_{n+1}$ is found equal to $T_{n}$. The idea is similar for $f \exists \mathcal{U} g$. We discover all of those transitions where $g$ is true in the source state. These transitions automatically satisfy $f \exists \mathcal{U} g$. Then we work our way backwards, constructing paths to this base set over which $f$ is always true.

The complete compositional model checking approach is summarized in Figure 6.

\section{Compositional Model Checking}

\section{Toolset}

The toolset is best illustrated by applying it to an example, in this case the $n$ task chain example given in section 2 . Consider the property mentioned in section 2 :

Whenever task $P_{i, i<n}$ has terminated, task $P_{i+1}$ will eventually terminate.

Or in CTL,

$$
F \equiv \bigwedge_{i=1}^{n-1} \operatorname{term}\left(P_{i}\right) \leadsto \operatorname{term}\left(P_{i+1}\right) .
$$

To show that $\left(P_{1}\left\|P_{2}\right\| \ldots \| P_{n}\right) \models F$, we divide this problem so that it can be attacked compositionally. Let $F_{i}$ be defined as follows:

$$
F_{i} \equiv \operatorname{term}\left(P_{i}\right) \sim \operatorname{term}\left(P_{i+1}\right)
$$

where $1 \leq i<n$. Hence, $F \equiv \wedge_{i=1}^{n-1} F_{i}$.

We use the following three properties of CTL to make deductions from the formulae checked by the model checker:

$$
\begin{array}{lll}
\mathcal{F} \leadsto \mathcal{G} & \forall \square(\mathcal{F} \Longrightarrow \forall \square \mathcal{G}) & \\
\mathcal{G} \leadsto \mathcal{H} & \mathcal{F} \leadsto \mathcal{H} & \text { True } \sim \mathcal{G} \\
\overline{\mathcal{F} \leadsto \mathcal{H}}(1) & \overline{\mathcal{F} \leadsto(\mathcal{G} \wedge \mathcal{H})}(2) & \overline{\mathcal{F} \leadsto \mathcal{G}} .(3)
\end{array}
$$

All three properties can be derived from the base rules for CTL. We can use property (1) to strengthen the antecedent 
1. FOR each Ada tasking program DO

(a) Translate the Ada task into a finite transition system.

(b) Remove transitions that are semantically inconsistent or unreachable.

2. SELECT a specification stated as a $\forall$ CTL sentence.

(a) Parse the selected sentence, negate it, and emit a postfix form $\exists$ CTL sentence.

3. WHILE there are any unchecked or uncomposed models DO

(a) Either CHOOSE an unchecked model, removing it from the set of models and

- Decide the subset of the selected model that (potentially) satisfies the sentence.

- Remove non-satisfying transitions, creating a new model.

(b) or CHOOSE some models, removing them from the set of models and

- Compose the models and refine the composition creating a new model.

(c) IF the new model is empty THEN RETURN True ELSE add the new model to the set of models.

4. RETURN False. (The remaining model contains the counter-examples.)

Figure 6: Compositional model checking procedure.

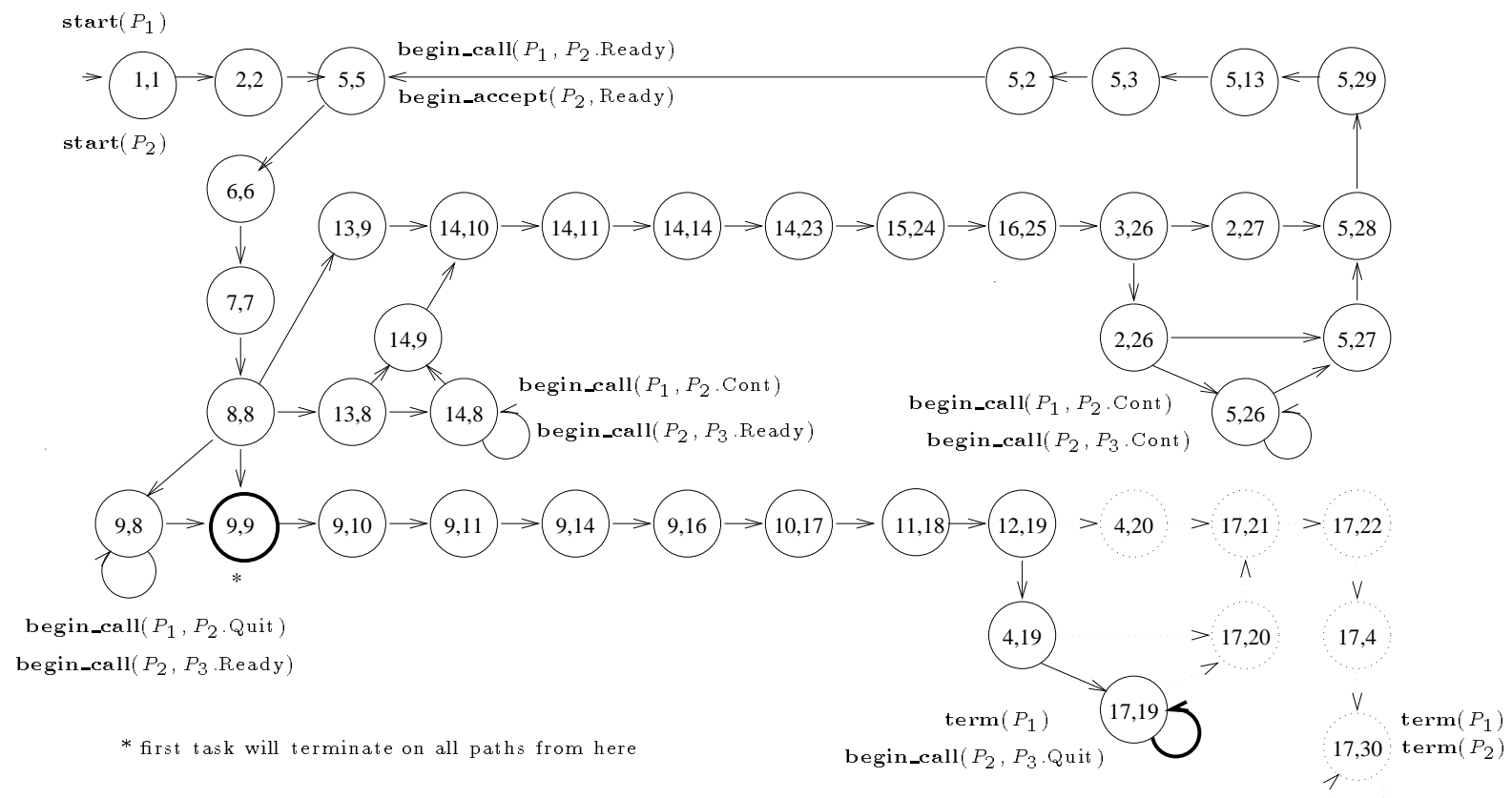

Figure 7: Composition of $P_{1}$ and $P_{2}$. 
in the implication if we are not able to prove the formula $\mathcal{F} \leadsto \mathcal{H}$ with the model checker. Assume that, we substitute $\mathcal{F} \wedge \mathcal{A}$ for $\mathcal{G}$ in property (1), where $\mathcal{A}$ is the strengthening assertion. If strengthening assertion $\mathcal{A}$ removes the transitions which create potential counter-examples, we will be able to prove the property $(\mathcal{F} \wedge \mathcal{A}) \leadsto \mathcal{H}$ using the model checker. If we can also prove $\mathcal{F} \leadsto(\mathcal{F} \wedge \mathcal{A})$, then using property (1) we can deduce $\mathcal{F} \leadsto \mathcal{H}$.

Steps of the analysis are summarized in Figure 8, where justification of each step is written in the right column. The lines which are marked "*" indicate proof obligations, i.e. formulae which are proved in following steps.

Step 1.1 : We note that the sentence $F_{1}$ only constrains tasks $P_{1}$ and $P_{2}$; many (possibly all) states of the other $n-2$ tasks might be consistent with both $F_{1}$ and $\neg F_{1}$. This fact naturally yields the following strategy: to first run $\operatorname{check}\left(P_{1} \| P_{2}, \neg F_{1}\right)$, and see if the result is $\emptyset$. If the returned set of states is non-empty, then it contains the potential counter-example to $F_{1}$.

In our case the result is nonempty. Figure 7 shows the transition system corresponding to $P_{1} \| P_{2}$ which has 43 states and 55 transitions. When we check $\neg F_{1}$ on $P_{1} \| P_{2}$ we get a minor reduction. The submodel returned by $\operatorname{check}\left(P_{1} \| P_{2}, \neg F_{1}\right)$ is drawn with solid lines in Figure 7 , and it has 37 states and 46 transitions. These may ultimately be shown to be unreachable behaviors, restricted by interactions with the other tasks. At this point they must be retained as "candidate" counter-examples, and they serve to guide the next steps in our analysis.

Consider the composite state $(9,9)$ (marked with * in Figure 7). The state corresponds to $P_{1}$ having signalled its readiness to terminate, after which $P_{2}$ will prepare to terminate as well. But this cannot happen until $P_{3}$ allows it, and so on. In fact, the self-loop on composite state $(17,19)$ is the critical transition for a counter-example, in that $\neg F_{1}$ holds if and only if the self-loop is taken infinitely often. The loop corresponds to $P_{3}$ 's refusal to accept $P_{2}$ 's call; if we can prove that $P_{3}$ accepts the call, then we will have no counter-example to $F_{1}$.

Now we can show how strengthening assertions can aid in the compositional approach. Consider property $F_{1}$ : “Whenever $P_{1}$ has terminated, $P_{2}$ will eventually terminate." We will strengthen it with $F_{1}^{\prime}$ : "Whenever $P_{1}$ has terminated and $P_{2}$ is not waiting for its calls to be accepted, $P_{2}$ will eventually terminate." $F_{1}^{\prime}$ can be stated as:

$$
F_{1}^{\prime} \equiv\left(\operatorname{term}\left(P_{1}\right) \wedge A_{2}\right) \leadsto \operatorname{term}\left(P_{2}\right)
$$

where

$$
\begin{aligned}
A_{2} \equiv & \neg \text { wait_call }\left(P_{2}, P_{3} . \text { Ready }\right) \wedge \\
& \neg \text { wait_call }\left(P_{2}, P_{3} . \text { Quit }\right) \wedge \\
& \neg \text { wait_call }\left(P_{2}, P_{3} . \text { Cont }\right) .
\end{aligned}
$$

Here, wait_call $\left(P_{i}, P_{i+1} . X\right)(X \in\{$ Ready, Quit, Cont $\})$ is defined as

$$
\begin{aligned}
\text { wait_call }\left(P_{i}, P_{i+1} . X\right) \equiv & \operatorname{begin\_ call}\left(P_{i}, P_{i+1} \cdot X\right) \wedge \\
& \neg \operatorname{begin\_ accept}\left(P_{i+1}, X\right) .
\end{aligned}
$$

So the deductive step which follows from property (1) is:

$$
\begin{aligned}
& \operatorname{term}\left(P_{1}\right) \leadsto\left(\operatorname{term}\left(P_{1}\right) \wedge A_{2}\right) \\
& F_{1}^{\prime} \equiv\left(\operatorname{term}\left(P_{1}\right) \wedge A_{2}\right) \leadsto \operatorname{term}\left(P_{2}\right) \\
& F_{1} \equiv \operatorname{term}\left(P_{1}\right) \leadsto \operatorname{term}\left(P_{2}\right)
\end{aligned}
$$

We call the model checker to verify $F_{1}^{\prime}$ on the composition $P_{1} \| P_{2}$, and as can be seen in Figure 9's dialog box, $\operatorname{check}\left(P_{1} \| P_{2}, \neg F_{1}^{\prime}\right)=\emptyset$; so $F_{1}^{\prime}$ holds.

Step 1.2 : Now we have to $\operatorname{prove} \operatorname{term}\left(P_{1}\right) \leadsto\left(\operatorname{term}\left(P_{1}\right) \wedge\right.$ $A_{2}$ ). To prove it we will use property (2) as follows:

$$
\begin{aligned}
& \forall \square\left(\operatorname{term}\left(P_{1}\right) \Longrightarrow \forall \square\left(\operatorname{term}\left(P_{1}\right)\right)\right) \\
& \frac{\operatorname{term}\left(P_{1}\right) \leadsto A_{2}}{\operatorname{term}\left(P_{1}\right) \leadsto\left(\operatorname{term}\left(P_{1}\right) \wedge A_{2}\right)}
\end{aligned}
$$

We check $\forall \square\left(\operatorname{term}\left(P_{1}\right) \Longrightarrow \forall \square\left(\operatorname{term}\left(P_{1}\right)\right)\right)$, and do not get any counter-examples, hence it holds. I.e., once $P_{1}$ terminates, it remains terminated.

Step 1.3 : Instead of proving $\operatorname{term}\left(P_{1}\right) \leadsto A_{2}$, we prove a stronger property: "All of $P_{2}$ 's calls are eventually accepted." That is, $A_{2}$ eventually holds. Formally, we want to prove the property $I_{2} \equiv$ True $\sim A_{2} \equiv \forall \square \forall \diamond A_{2}$. If we can prove this property, then $\operatorname{term}\left(P_{1}\right) \leadsto A_{2}$ is also true, since the consequent of the implication is always true (property (3)). Then using property (2) we can deduce $\operatorname{term}\left(P_{1}\right) \leadsto\left(\operatorname{term}\left(P_{1}\right) \wedge A_{2}\right)$, as shown above. This will also allow us to deduce $F_{1} \equiv \operatorname{term}\left(P_{1}\right) \sim \operatorname{term}\left(P_{2}\right)$ using property (1) as explained above.

If we try to prove the property $I_{2}$ on the composition $P_{1} \| P_{2}$ we will not be successful because $I_{2}$ is related to the behavior of $P_{3}$. So, as the next step we compose $P_{2}$ and $P_{3}$ on which we will try to prove $F_{2}$ and $I_{2}$.

Steps 2.1 - 2.3 : These steps are identical to steps 1.1 1.3. We first check $F_{2}^{\prime}$ on $P_{2} \| P_{3}$ and get an empty transition system as the result, so $F_{2}^{\prime}$ holds. If we can show that $I_{3}$ 


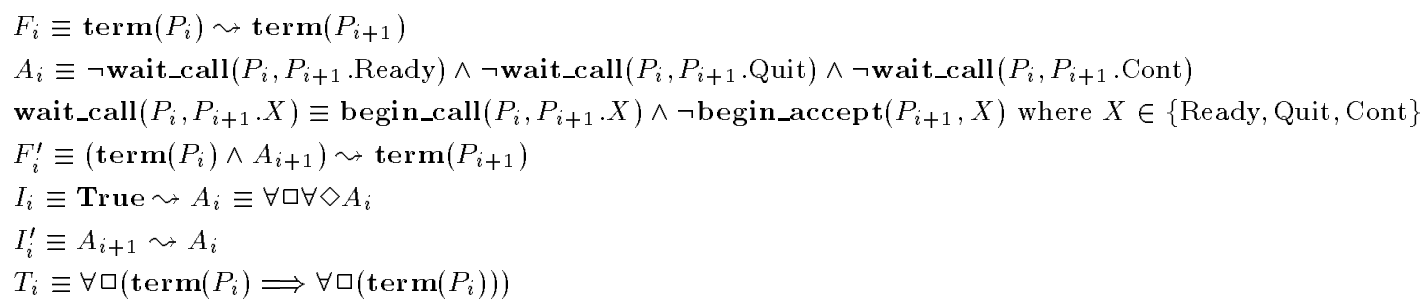

FORMULA

$1.1 \quad \operatorname{term}\left(P_{1}\right) \leadsto\left(\operatorname{term}\left(P_{1}\right) \wedge A_{2}\right)$

$F_{1}^{\prime} \equiv\left(\operatorname{term}\left(P_{1}\right) \wedge A_{2}\right) \leadsto \operatorname{term}\left(P_{2}\right)$

$\overline{F_{1} \equiv \operatorname{term}\left(P_{1}\right) \leadsto \operatorname{term}\left(P_{2}\right)}$

$1.2 \quad T_{1} \equiv \forall \square\left(\operatorname{term}\left(P_{1}\right) \Longrightarrow \forall \square\left(\operatorname{term}\left(P_{1}\right)\right)\right)$

$\operatorname{term}\left(P_{1}\right) \leadsto A_{2}$

$\overline{\operatorname{term}\left(P_{1}\right) \leadsto\left(\operatorname{term}\left(P_{1}\right) \wedge A_{2}\right)}$

1.3

$\frac{I_{2} \equiv \text { True } \leadsto A_{2}}{\operatorname{Term}\left(P_{1}\right) \leadsto A_{2}}$

Remaining proof obligations from step $1: I_{2} \equiv$ True $\sim A_{2}$

$2.1 \quad \operatorname{term}\left(P_{2}\right) \leadsto\left(\operatorname{term}\left(P_{2}\right) \wedge A_{3}\right)$

$F_{2}^{\prime} \equiv\left(\operatorname{term}\left(P_{2}\right) \wedge A_{3}\right) \leadsto \operatorname{term}\left(P_{3}\right)$

$\overline{F_{2} \equiv \operatorname{term}\left(P_{2}\right) \leadsto \operatorname{term}\left(P_{3}\right)}$

2.2 $T_{2} \equiv \forall \square\left(\operatorname{term}\left(P_{2}\right) \Longrightarrow \forall \square\left(\operatorname{term}\left(P_{2}\right)\right)\right)$

$\operatorname{term}\left(P_{2}\right) \leadsto A_{3}$

$\overline{\operatorname{term}\left(P_{2}\right) \leadsto\left(\operatorname{term}\left(P_{2}\right) \wedge A_{3}\right)}$

$2.3 \quad \frac{I_{3} \equiv \operatorname{True} \leadsto A_{3}}{\operatorname{term}\left(P_{2}\right) \sim A_{3}}$

2.4 $I_{3} \equiv$ True $\sim A_{3}$

$I_{2}^{\prime} \equiv A_{3} \sim A_{2}$

$\overline{I_{2}} \equiv$ True $\sim A_{2}$

Remaining proof obligations from step $2: I_{3} \equiv$ True $\leadsto A_{3}$

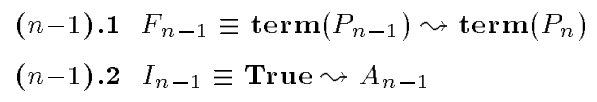

$(n-1) .1 \quad F_{n-1} \equiv \operatorname{term}\left(P_{n-1}\right) \leadsto \operatorname{term}\left(P_{n}\right)$

\section{J USTIFICATION}

* (Proved in step 1.2)

$\operatorname{check}\left(P_{1} \| P_{2}, \neg F_{1}^{\prime}\right)=\emptyset$

Property (1)

$\operatorname{check}\left(P_{1}, \neg T_{1}\right)=\emptyset$
$*($ Proved in step 1.3$)$

Property (2)

* (Proved in step 2.4)

Property (3)

* (Proved in step 2.2)

$\operatorname{check}\left(P_{2} \| P_{3}, \neg F_{2}^{\prime}\right)=\emptyset$

Property (1)

*heck $\left(P_{2}, \neg T_{2}\right)=\emptyset$

Property (2)

* (Proved in step 3.4)

Property (3)

* (Proved in step 3.4)

$\operatorname{check}\left(P_{2} \| P_{3}, \neg I_{2}^{\prime}\right)=\emptyset$

Property (1)

Figure 8: Summary of the analysis. 


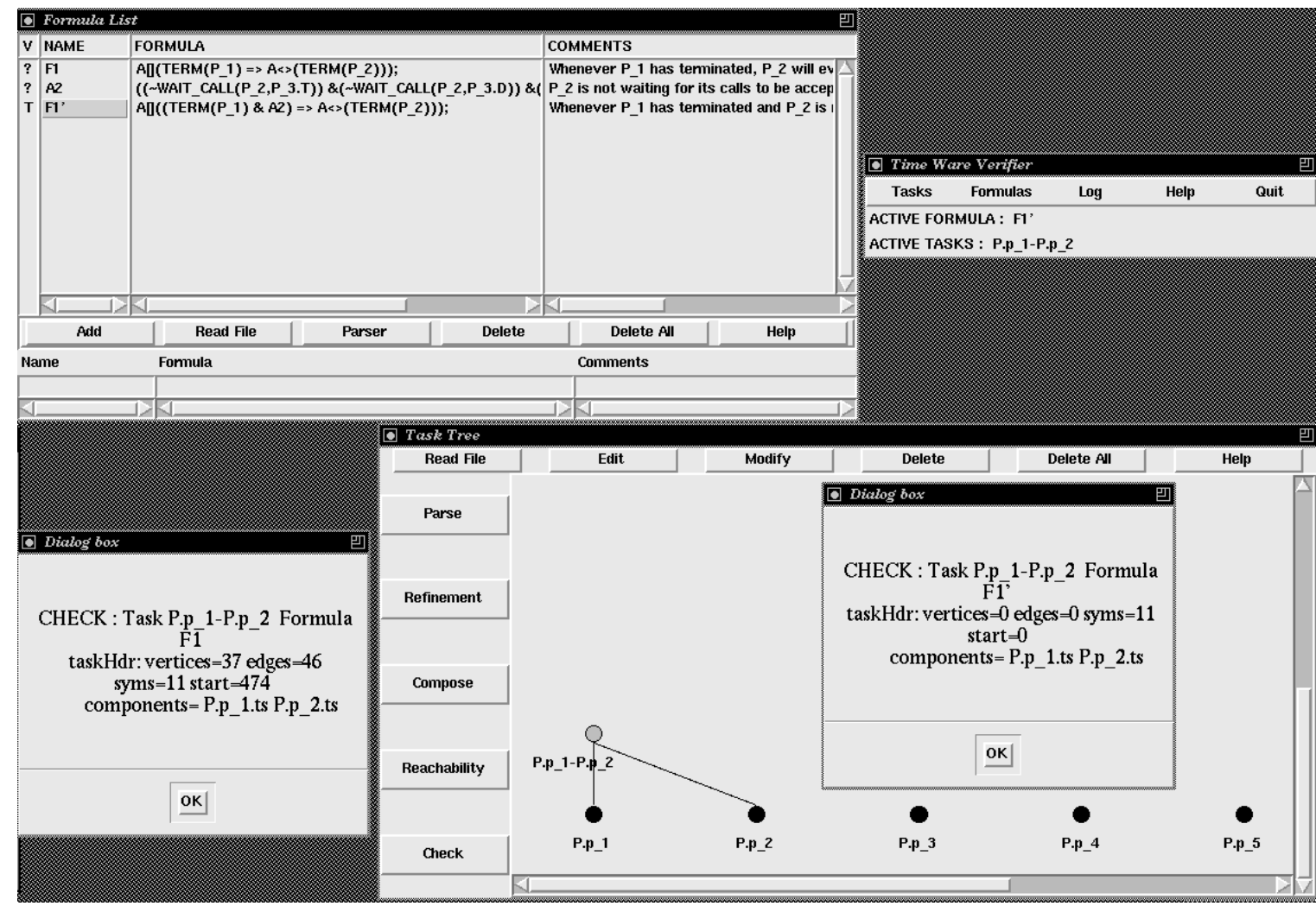

Figure 9: Results of $\operatorname{check}\left(P_{1} \| P_{2}, \neg F_{1}\right)$ and $\operatorname{check}\left(P_{1} \| P_{2}, \neg F_{1}^{\prime}\right)$.

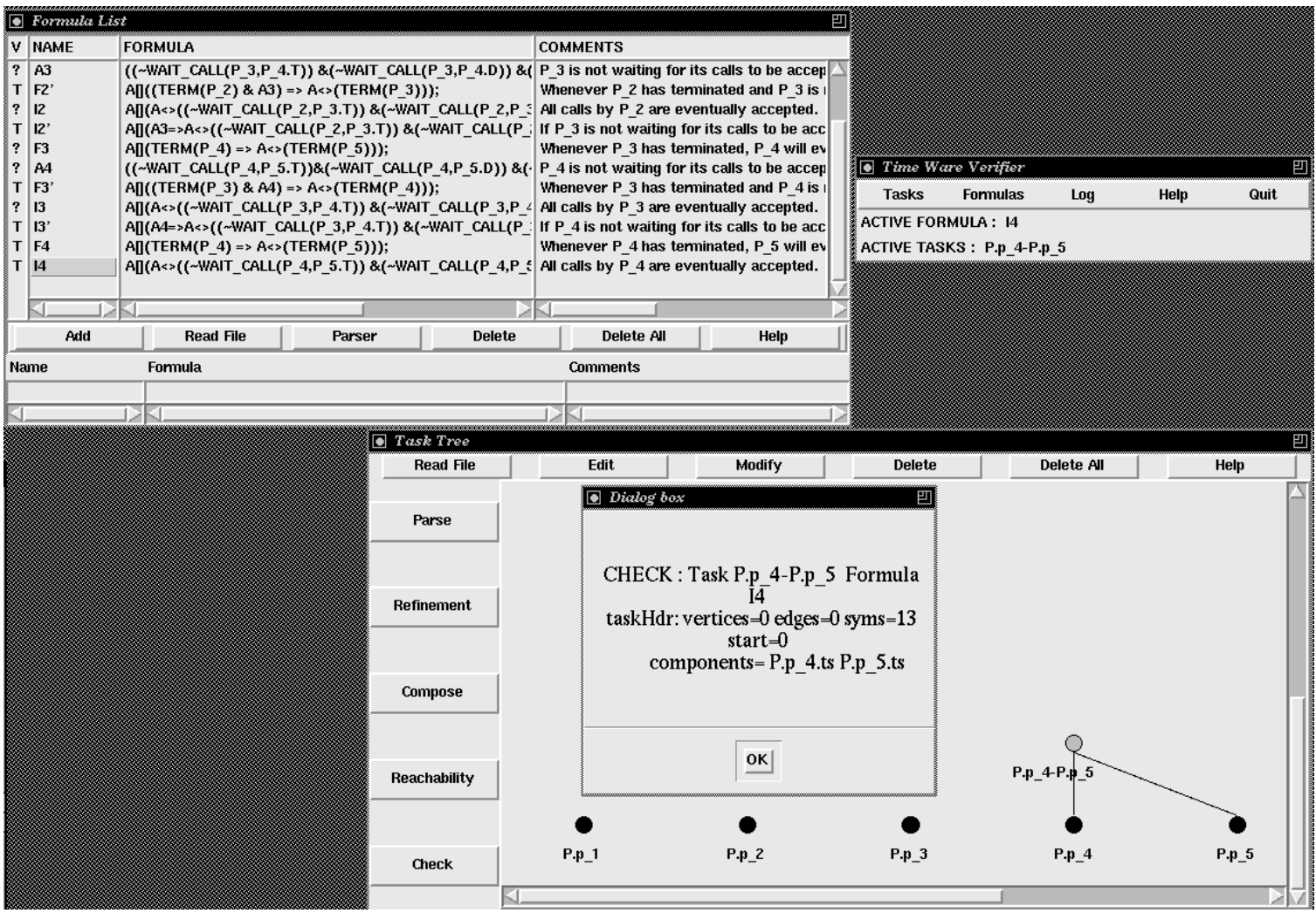

Figure 10: Result of $\operatorname{check}\left(P_{4} \| P_{5}, \neg I_{4}\right)$. 
holds, then from $F_{2}^{\prime}$ and $I_{3}$ we can deduce $F_{2}$, just as we did for $F_{1}$. So $I_{3}$ is the proof obligation in these steps.

Step 2.4 : Now we have to prove the proof obligation from the previous step, i.e. $I_{2}$. If we try to prove the property $I_{2}$ on $P_{2} \| P_{3}$ we will have the same problem we had for $F_{1}$. So we use the strengthening argument again. Instead of proving $I_{2}$ we try to prove $I_{2}^{\prime} \equiv A_{3} \leadsto A_{2}$. Then using property (1):

$$
\begin{aligned}
& I_{3} \equiv \text { True } \leadsto A_{3} \\
& I_{2}^{\prime} \equiv A_{3} \leadsto A_{2} \\
& \overline{I_{2} \equiv \text { True } \leadsto A_{2}}
\end{aligned}
$$

we deduce $I_{2}$. When we call $\operatorname{check}\left(P_{2} \| P_{3}, \neg I_{2}^{\prime}\right)$, we get $\emptyset$ as the result, so $I_{2}^{\prime}$ holds.

Steps $3-(n-2)$ : If we continue to repeat the operations discussed above we will get the same results until the $n-1$ st step, since the intermediate $n-2$ tasks are identical.

Step $(n-1):$ In the $(n-1)$ st step we notice that we do not need the strengthening assertions anymore! The reason is that we reached the end of the task chain, and $P_{n}$ does not have to wait for any other task. It will accept all the calls from $P_{n-1}$. So we first call $\operatorname{check}\left(P_{n-1} \| P_{n}, \neg F_{n-1}\right)$ and get $\emptyset$ as the result, so $F_{n-1}$ holds. Now we have to prove the last proof obligation from the previous step, i.e. $I_{n-1}$, which will complete the proof. Figure 10 shows the result of $\operatorname{check}\left(P_{n-1} \| P_{n}, \neg I_{n-1}\right)$ for $n=5$, and it is $\emptyset$, so $I_{n-1}$ is satisfied, hence the proof is complete.

Note that, the proof given above is a semi-automated process. We designed the structure of the proof by examining the counter-example output by the model checker. After coming up with the strengthening assertions (which still needs human interaction) we used the model checker to verify the formulae in each step. We did not have to deal with the details involved in verifying these formulae, which may be very tedious.

\section{$5 \quad$ Results and Future Work}

While the introduction of our compositional approach has not increased the worst case complexity, if the worst case is realized then we haven't gained anything over the traditional approach. We believe, however, that for many systems and properties our approach can do dramatically better than the traditional approach.

Figure 11 illustrates the tremendous savings that can sometimes be provided by our compositional approach. Here we are using the total number of transitions that must be

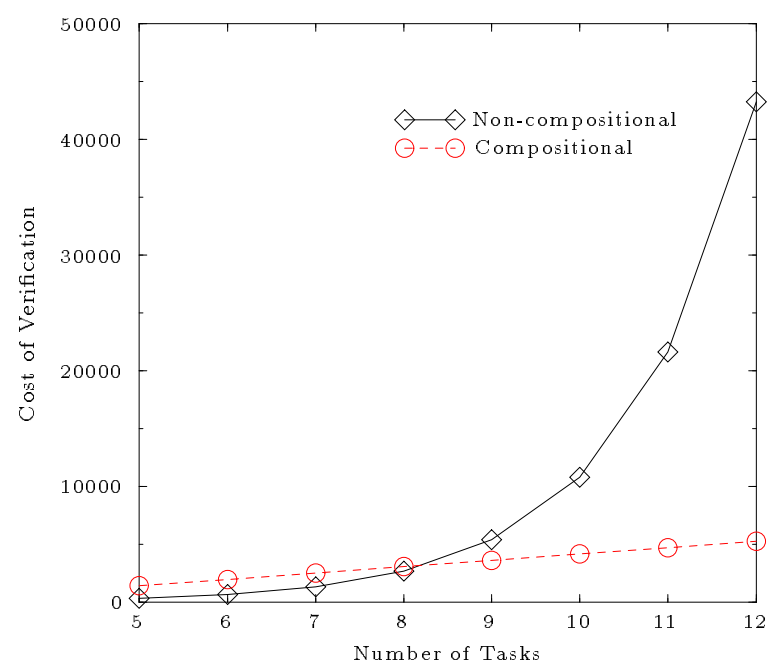

Figure 11: Cost of compositional and non-compositional model-checks of $n$ task chain.

examined and/or composed as the cost metric. In the case of the $n$ task chain, when following the traditional approach our composition tool ran out of memory even for small values of $n$. But with our compositional method we never need to create such large $n$-way compositions to perform the verification, for any $n$.

Our experiments have shown that there can be a large variance in the total cost of checking. The variance is caused by differences in models, formulas, and order of operations. E.g., given two compositional checks of the same sentence, and on the same components, the total cost can be orders of magnitude apart! As part of our on-going work we are seeking to develop heuristics for generating compositional model checking schedules that, given a sentence and a model, would suggest:

- Which sentence(s) to check: Should we break the original sentence down into a set of conjuncts or disjuncts, and then check each new sentence individually?

- The order of checks and compositions : Which models should be checked first? Which models should be checked last? Which models should we compose before checking?

- Adjustments to the schedule based upon feedback : What strengthening assertions are suggested by the potential counter-examples identified by the model checker? If checking a model yields a very large reduction, then should we immediately compose the result 
with another model: If so, which model? If we see our compositions are blowing up, should we backup and take a different scheduling path?

Even if scheduling guidance is not incorporated into the tool itself, we see the development of some rules of thumb as being critical to the practical application of a compositional approach.

As part of our future work we intend to add real-time modelling capabilities. While there has been recent research in real-time model checking (see [1, 12]), there has been very little exploration using a compositional framework. The addition of time makes this a significantly more complex problem.

\section{Conclusions}

We have developed an automated approach to verification that is compositional. As we have demonstrated with an example, our approach enables us to pursue verification efforts that cannot be handled by the traditional model checking approaches. An additional benefit of our method is that in those cases where the property being analyzed does not hold we generate the execution paths that serve as counterexamples. We have implemented an automated tool-set that supports our approach and intend to expand both the toolset and theory with heuristics for selecting compositional verification strategies and support for real-time properties.

\section{References}

[1] R. Alur, C. Courcoubetis, T. A. Henzinger, and P. Ho. Hybrid automata: an algorithmic approach to the specification and verification of hybrid systems. Proceedings of the Workshop on Theory of Hybrid Systems, 1992.

[2] J. R. Burch, E. M. Clarke, K. L. McMillan, D. L. Dill, and L. H. Hwang. Symbolic model checking: $10^{20}$ states and beyond. Proceedings of the Fifth Annual Symposium on Logic in Computer Science, 428-439, 1990.

[3] M. Chiodo, T. R. Shiple, A. Sangiovanni-Vincentelli, and R. K.Brayton. Automatic reduction in CTL compositional model checking. Proceedings CAV'92, LNCS 663, 234-247, June 1992 .

[4] E. M. Clarke, E. A. Emerson, and A. P. Sistla. Automatic verification of finite-state concurrent systems using temporal logic specifications. ACM Transactions on Programming Languages and Systems, 8(2):244-263, April 1986

[5] S. C. Cheung and J. Kramer. Enhancing Compositional Reachability Analysis with Context Constraints. Proceedings FSE'93, ACM SIGSOFT, 115-125, December 1993
[6] R. Cleaveland. Tableau-based model checking in the propositional mu-calculus. Acta Informatica, 27:725-747, 1990

[7] R. Cleaveland, J. Parrow, and B. Steffen. The Concurrency Workbench. Proceedings of the Workshop on Automatic Verification Methods for Finite-State Systems, LNCS 407, 24-37, 1990.

[8] E. M. Clarke, D. E. Long, and K. L. McMillan. Compositional model checking. Proceedings of the Fourth Annual Symposium on Logic in Computer Science, 464-475, June 1989.

[9] E. A. Emerson and J. Y. Halpern. 'Sometimes' and 'not never' revisited: on branching versus linear time temporal logic. Journal of the ACM, 33(1):151-178, January 1986.

[10] E. A. Emerson and C. Lei. Efficient model checking in fragments of the propositional mu-calculus. Proceedings of Symposium on Logic in Computer Science, 267-278, 1986

[11] J. Fischer and R. Gerber. Compositional Model Checking of Ada Tasking Programs Proceedings COMPASS'94. 1994.

[12] N. Halbwachs. Delay analysis in synchronous programs CAV93, LNCS 697. 1993.

[13] R. J. van Glabbeek. The linear time - branching time spectrum. CONCUR90, LNCS 458,1990

[14] R. Gerber and I. Lee. A Layered Approach to Automating the Verification of Real-Time Systems. IEEE Trans. on Software Eng., 18(9):768-784, 1992 .

[15] O. Lichtenstein and A. Pnueli. Checking that finite state concurrent programs satisfy their linear specifications. 1984.

[16] J. S. Ostroff. Survey of formal methods for the specification and design of real-time systems. Draft for IEEE Press book "Tutorial on Specification of Time", 1992.

[17] C. Stirling and D. Walker. CCS, liveness, and local model checking in the linear time mu-calculus. Automatic Verification Methods for Finite State Systems, LNCS 407, 1989.

[18] W. J. Yeh and M. Young. Compositional Reachability Analysis Using Process Algebra. Proceedings TAV4, ACM SIGSOFT, 49-59, October 1991. 